\title{
Aluminum Alloy Material Structure Impact Localization by Using FBG Sensors
}

\author{
Xiubin $\mathrm{ZHU}^{*}$ \\ School of Electric and Electronic Engineering, Zibo Vocational Institute, Zibo, 255314, China \\ *Corresponding Author: Xiubin ZHU_ E-mail: zibozhuxiubin@163.com
}

\begin{abstract}
The aluminum alloy structure impact localization system by using fiber Bragg grating (FBG) sensors and impact localization algorithm was investigated. A four-FBG sensing network was established. And the power intensity demodulation method was initialized employing the narrow-band tunable laser. The wavelet transform was used to weaken the impact signal noise. And the impact signal time difference was extracted to build the time difference localization algorithm. At last, a fiber Bragg grating impact localization system was established and experimentally verified. The experimental results showed that in the aluminum alloy plate with the $500 \mathrm{~mm} * 500 \mathrm{~mm} * 2 \mathrm{~mm}$ test area, the maximum and average impact abscissa localization errors were $11 \mathrm{~mm}$ and $6.25 \mathrm{~mm}$, and the maximum and average impact ordinate localization errors were $9 \mathrm{~mm}$ and $4.25 \mathrm{~mm}$, respectively. The fiber Bragg grating sensors and demodulation system are feasible to realize the aviation aluminum alloy material structure impact localization. The research results provide a reliable method for the aluminum alloy material structure impact localization.
\end{abstract}

Keywords: Fiber Bragg grating, aluminum alloy plate impact localization, time difference localization, wavelet transform

Citation: Xiubin ZHU, “Aluminum Alloy Material Structure Impact Localization by Using FBG Sensors,” Photonic Sensors, 2014, 4(4): $344-348$

\section{Introduction}

The aircraft often suffers impact in service, such as stone impact, bird impact, and hail impact. These impacts would lead to part of the structure damaged or cause the crash of the aircraft, which bring disastrous consequences [1]. Therefore, with the aid of the installed corresponding sensor in the aircraft vulnerable structure, the constructed sensor network, and the on-line monitoring impact events, identifying the impact position is an important part to guarantee the safe operation of the aircraft.

The impact signal which is produced by the object impact aviation material structure belongs to the sudden acoustic emission signal, so the acoustic emission (AE) source localization technology could be used for the aviation material structure impact research [2]. In general, the $\mathrm{AE}$ localization technology often uses the time difference localization method. Jie Huang et al. [3] achieved the impact localization on a $300 \mathrm{~mm} * 800 \mathrm{~mm} * 2 \mathrm{~mm}$ thick aluminum plate within $10 \mathrm{~mm}$ by using four polyvinylidene fluoride sensors. In their research, they realized the impact localization by the measurements of the distance between each sensor, the impact signal wave velocity, and the impact signal arrival time difference. Through installing a series of sensors in the aircraft structure, 
constructing the sensor network, and then extracting time difference according to the impact signal propagation time of each sensor, the time difference localization algorithm was adopted to realize the impact localization. This approach has achieved good results by using the piezoelectric sensors [4]. The requirements of aerospace's structural health monitoring, such as light sensor, demodulation equipment's quality and small volume, no destruction of the material structure, and resistance to electromagnetic interference, are difficult to meet. In recent years, the fiber Bragg grating (FBG) sensor with its advantages of the small size, light weight, high sensitivity, strong electromagnetic interference, corrosion resistance can be used in the sensor network and applied in the aviation field $[5,6]$. So we used FBG sensor in the research.

Based on the above analysis, the FBG sensors can be used to construct the sensor network, monitor impact events on-line, and realize the aluminum alloy material structure impact localization. Wavelet transformation $[7,8]$ can be used to weaken the noise contained in the impact signals. And then the time difference localization algorithm was used to realize the impact localization. The research provided a reliable method for the aluminum alloy material structure impact localization.

\section{Impact localization algorithms}

In the experiments, the system noise and the external environment disturbance made the impact signal contain noise. The signal noise directly affects the accuracy of the impact localization, so it is necessary to remove the noise.

\subsection{Weaken signal noise by wavelet transformation}

The wavelet transformation was used to weaken the signal noise, as follows:

(1) Decomposition process. Select a kind of wavelet and then obtain the signal as $N$-layer wavelet decomposition.

(2) Action threshold process. Choose a threshold for every decomposed layer coefficient and then use the threshold as the processed coefficient.

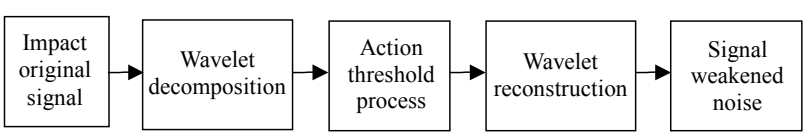

Fig. 1 Processes of the used wavelet transform to weaken the signal noise.

(3) Reconstruction process. Signal noise reduction is performed by the wavelet reconstruction of the processed coefficient.

Thus, we can weaken the impact signal noise by using the wavelet transformation based on the above processes.

\subsection{Time difference localization algorithm}

The principle of the time difference localization algorithm is as follows. Through installing a series of sensors in the aircraft structure, constructing the sensor network, and then extracting time difference according to the impact signal propagation time of each sensor, the time difference localization algorithm was adopted to realize the impact localization. FBG sensors named FBG1, FBG2, FBG3, and FBG4 were placed in the square structure, as shown in Fig.2.

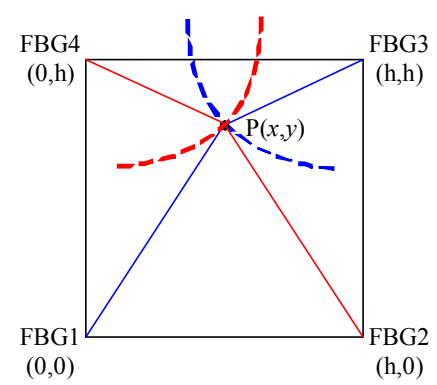

Fig. 2 Schematic diagram of the time difference extraction algorithm.

Define the arrival time of the impact signal generated by the impact point $\mathrm{P}$ reaching FBG1, FBG2, FBG3, and FBG4 as $t_{1}, t_{2}, t_{3}$ and $t_{4}$, then the signal time differences $t_{x}$ and $t_{y}$ are as follows:

$$
t_{x}=t_{1}-t_{3}, t_{y}=t_{2}-t_{4} .
$$

And then, the impact signal propagation distances are as follows:

$$
l_{x}=v t_{x}, l_{y}=v t_{y}
$$


where $v$ is the impact signal propagation velocity. Then, the impact point $\mathrm{P}$ is the intersection of two hyperbolas which focuses at FBG1, FBG3 and FBG2, FBG4, respectively. Thus, the coordinates of the impact point $\mathrm{P}$ can be obtained according the following equations:

$$
\left\{\begin{array}{l}
\sqrt{x^{2}+y^{2}}-\sqrt{(x-h)^{2}+(y-h)^{2}}=l_{x} \\
\sqrt{(x-h)^{2}+y^{2}}-\sqrt{x^{2}+(y-h)^{2}}=l_{y}
\end{array} .\right.
$$

According to (3), we can obtain the coordinates of the impact point $P$, thus we complete the aluminum alloy structure impact localization based on the above algorithm.

\section{Impact localization system setup}

\subsection{FBG strain measurement principle}

The principle of FBG sensing is as follows:

$$
\lambda_{B}=2 n \Lambda
$$

where $\lambda_{B}$ is the Bragg wavelength, $n$ is the effective refractive index of the FBG, and $\Lambda$ is grating period. The FBG is sensitive to the temperature and strain at the same time. Only considering the influence of the strain, according to (4), we can get the relationship between the FBG wavelength change and strain as follows:

$$
\frac{\Delta \lambda_{B}}{\lambda_{B}}=\left(1-P_{e}\right) \cdot \varepsilon
$$

where $\Delta \lambda_{B}$ is the reflection wavelength change caused by the strain, $P_{e}$ is the effective light function, $\varepsilon$ is the strain. Equation (5) shows that when the impact aluminum alloy plate produces the strain, the wavelength of the FBG will change, so we can use FBG sensors to monitor the impact signal.

\subsection{Experiment system setup}

The FBG impact localization experiment system was mainly composed of the tunable laser, photoelectric detector, high-speed data acquisition equipment, aluminum alloy plate, and FBG sensors. The photoelectric detector was New Focus model
2053 with the band pass frequency of $1 \mathrm{kHz}-$ $100 \mathrm{kHz}$ and the magnification of 100 . The sampling frequency was $5 \mathrm{MHz}$. The aluminum alloy plate was 6061-T6 with the Young's modulus of $69 \mathrm{GPa}$, Poisson's ratio of 0.33 , density of $2700 \mathrm{~kg} / \mathrm{m}^{2}$, and size of $600 \mathrm{~mm} * 600 \mathrm{~mm} * 2 \mathrm{~mm}$. The aluminum alloy plate edges were clamped on the experimental bench with the area of $500 \mathrm{~mm} * 500 \mathrm{~mm} * 2 \mathrm{~mm}$ on the aluminum alloy plate as the experimental area. Four FBG sensors were pasted on the corners of the experimental area, and the wavelengths of FBG1, FBG2, FBG3, and FBG4 were $1562.589 \mathrm{~nm}$, $1562.590 \mathrm{~nm}, 1562.587 \mathrm{~nm}$, and $1562.590 \mathrm{~nm}$, respectively. And their location coordinates were $(0 \mathrm{~mm}, 0 \mathrm{~mm}),(500 \mathrm{~mm}, 0 \mathrm{~mm}),(500 \mathrm{~mm}, 500 \mathrm{~mm})$, and $(0 \mathrm{~mm}, 500 \mathrm{~mm})$, respectively. The steel ball was selected as the impact device with the weight of $25 \mathrm{~g}$. The ball impacted the aluminum alloy plate in the form of the free fall. The tunable laser was Santec tunable semiconductor laser TSL-510 with the light tuning range of $1560 \mathrm{~nm}$ to $1680 \mathrm{~nm}$, the maximum laser power of up to $10 \mathrm{~mW}$, and spectrum full width at half maximum of less than $1 \mathrm{pm}$.

The fundamental of the high-speed demodulation based on the tunable laser TSL-510 is as follows. Because the distribution function of the FBG reflected light intensity can be expressed as the Gaussian function, the edges of the FBG reflected spectrum has a small range approximately equal to the linear change. If the central wavelength of the tunable laser is tuned in this linear range, there is a linear relationship between the reflected light intensity and FBG wavelength:

$$
\varepsilon=\frac{1}{B \lambda_{B}\left(1-P_{e}\right)} \cdot \frac{\Delta P}{P}
$$

where $B$ is the linear range slope of the FBG reflection spectrum, $P$ is the light intensity, and $\Delta P$ is the variation of the light intensity. Therefore, when the acoustic emission signal propagates in the structure, the wavelength of the FBG pasted in the structure will be changed. And because the central wavelength of the tunable laser is constant, the FBG 
wavelength change will cause the change in the FBG reflected light intensity, thus we can realize the acoustic emission signal dynamic demodulation based on (6). The narrow line width characteristic of the tunable laser ensures that its detection response has the high sensitivity. In the experiments, when pasted on the aluminum alloy plate, all FBG sensors were attached to a certain prestress to keep the grating wavelength at $1562.600 \pm 0.001 \mathrm{~nm}$. The wavelength of the tunable laser was set at $1562.615 \mathrm{~nm}$, and the output power was set at $1 \mathrm{~mW}$. Besides, we selected a steel ball in the form of impact on the aluminum alloy plate to generate the acoustic emission signal. The FBG impact localization experimental systems are shown in Figs. 3 and 4.

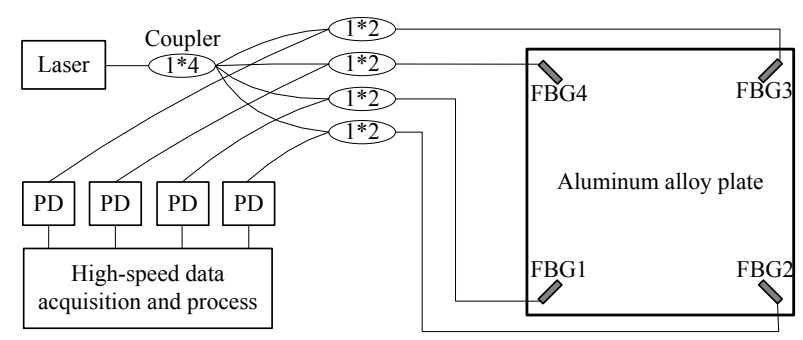

Fig. 3 FBG impact localization experimental system.

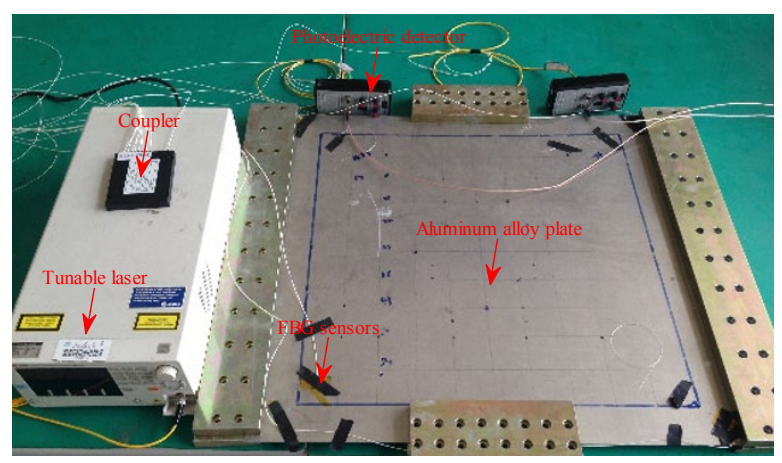

Fig. 4 Experimental system.

\section{Impact localization experiment and result analysis}

\subsection{Signal time difference extraction}

In this section, we will discuss how to explore the signal time difference extraction method by impacting the aluminum alloy plates at the point $(200 \mathrm{~mm}, 200 \mathrm{~mm})$. The monitoring impact signals of FBG1 and FBG3 sensors are shown in Fig. 5. In aluminum alloy materials, there are several propagation modes with different frequency bands in an impact wave. Thus, for the proper determination of the arrival time difference, the arrival time has to be measured from the leading waves or similar frequency portions of each sensor signal. In order to choose the specific propagation mode, which appropriately reflects the arrival moment of an impact wave, a wavelet transformation is applied to the obtained signals. In this study, the Daubechies 4 ( $\mathrm{db}$ 4) wavelet functions were adopted to process 4-scale decomposition. Among the decomposed wavelet portions, the A4 portion had the low noise level and could properly indicate the arrival time of the original signal as shown in Fig.6. According to Fig. 6, the selected threshold value was $0.04 \mathrm{~V}$, and then we calculated the signal time difference as $-57.6 \mathrm{~ns}$.
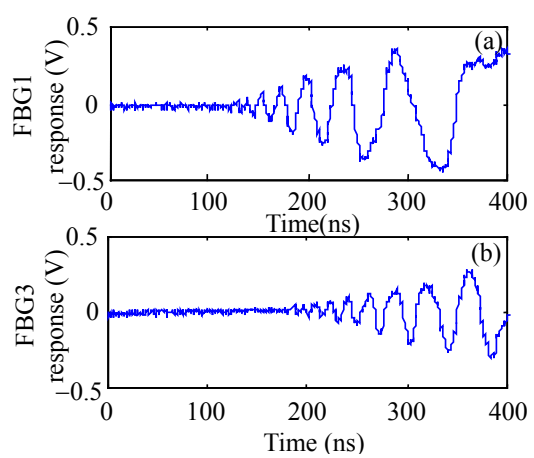

Fig. 5 Original signals of FBG1 and FBG3.
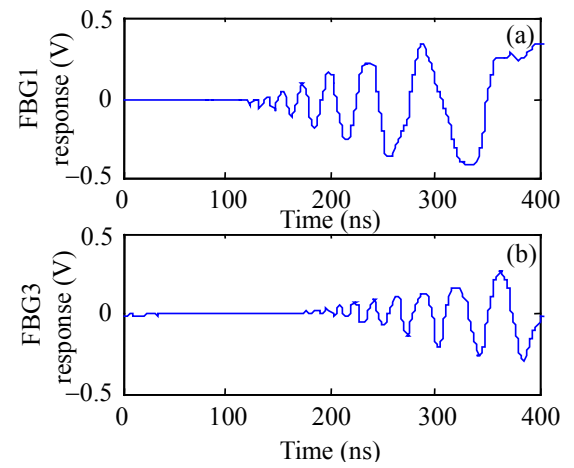

Fig. 6 Signals of FBG1 and FBG3 after weakened noises.

\subsection{Impact localization}

Before the impact location experiment, we need to determine the propagation velocity of the impact 
signal in the aluminum alloy structure. Because the aluminum alloy is an isotropic material, the signal propagation velocity in the aluminum alloy structure remains the same. Thus, we impacted the aluminum alloy plates at the point $(250 \mathrm{~mm}, 400 \mathrm{~mm})$ and calculated the signal propagation velocity. Based on the above time difference extraction method, we calculated the signal time difference as $93.2 \mathrm{~ns}$ and then the signal propagation velocity in the aluminum alloy structure as $2172.112 \mathrm{~m} / \mathrm{s}$ in this experimental environment.

On the basis of the signal propagation velocity of $2172.112 \mathrm{~m} / \mathrm{s}$, we selected any four points on the aluminum alloy plate and then used the time difference localization algorithm to localize the impact location. The results are shown in Table 1. From Table 1, we found that the maximum and average impact abscissa localization errors were $11 \mathrm{~mm}$ and $6.25 \mathrm{~mm}$, and the maximum and average impact ordinate localization errors were $9 \mathrm{~mm}$ and $4.25 \mathrm{~mm}$. Therefore, the aluminum alloy structure impact localization system by using FBG sensors had the high precision. So using FBG sensors to complete the aluminum alloy structure impact localization is feasible.

Table 1 Results of the impact localization.

\begin{tabular}{cccc}
\hline $\begin{array}{c}\text { Impact points } \\
(\mathrm{mm}, \mathrm{mm})\end{array}$ & $\begin{array}{c}\text { Localization points } \\
(\mathrm{mm}, \mathrm{mm})\end{array}$ & $\begin{array}{c}\text { Abscissa error } \\
(\mathrm{mm})\end{array}$ & $\begin{array}{c}\text { Ordinate error } \\
(\mathrm{mm})\end{array}$ \\
\hline$(150,400)$ & $(146,409)$ & -4 & 9 \\
$(250,350)$ & $(250,353)$ & 0 & 3 \\
$(175,300)$ & $(165,304)$ & -10 & 4 \\
$(200,200)$ & $(211,201)$ & 11 & 1 \\
\hline
\end{tabular}

\section{Conclusions}

We used FBG sensors to complete the aluminum alloy structure impact localization. Firstly, the wavelet transformation was used to weaken the signal noise. And then the signal time difference was extracted to complete the time difference localization algorithm. Finally, the FBG impact localization system was built, and the experimental verification was performed. The experimental results showed that the maximum and average impact abscissa localization errors were $11 \mathrm{~mm}$ and $6.25 \mathrm{~mm}$, and the maximum and average impact ordinate localization errors were $9 \mathrm{~mm}$ and $4.25 \mathrm{~mm}$, which can meet the practical application needs. So using the fiber Bragg grating sensors to realize the aviation aluminum alloy material structure impact localization is feasible.

Open Access This article is distributed under the terms of the Creative Commons Attribution License which permits any use, distribution, and reproduction in any medium, provided the original author(s) and source are credited.

\section{References}

[1] W. L. Zhou, "Research on impact load and damage identification methods for piezoelectric smart structures," Ph.D. dissertation, Nanjing University of Aeronautics and Astronautics, Nanjing, China, 2004.

[2] G. T. Shen, R. S. Geng, and S. F. Liu, "Acoustic emission source location," Nondestructive Testing, 2002, 24(3): 114-117.

[3] J. Huang, X. Z. Huang, and J. Y. Luo, "Localization technique for space debris impacted spacecraft with PVDF sensor," Journal of Astronautics, 2012, 33(9): 1341-1346.

[4] D. Liang, S. F. Yuan, and H. B. Sun, "Precise impact positioning method based on aerospace structure multi-agent coordination," Journal of Nanjing University of Aeronautics \& Astronautics, 2011, 43(2): 191-197.

[5] J. Y. Lu, B. F. Wang, and D. K. Liang, "Identification of impact location by using FBG based on wavelet packet feature extraction and SVR," Optics and , 2012, 20(2): 712-714.

[6] B. W. Jang, Y. G. Lee, J. H. Kim, Y. Y. Kim, and C. G. Kim, "Real-time impact identification algorithm for composite structures using fiber Bragg grating sensors," Structural Control and Health Monitoring, 2012, 19(7): 580-591.

[7] Y. H. Fang, C. Kong, T. G. Lan, W. Xiong, D. M. Dong, and D. C. Li, "Denoising and baseline correction of spectrum by wavelet transform," Optics and Precision Engineering, 2006, 14(6): 1088-1092.

[8] S. G. Mallat, "A theory for multiresolution signal decomposition: the wavelet representation," IEEE Transactions on Pattern Analysis and Machine Intelligence, 1989, 11(7): 647-693. 